\title{
Numerical Modelling of Stress and Strain Evolution during Solidification of a Single Crystal Superalloy
}

\section{Chinnapat Panwisawas ${ }^{1, a}$, Jean-Christophe Gebelin ${ }^{2, b}$, Nils Warnken ${ }^{3, c}$, Robert W Broomfield ${ }^{4, d}$ and Roger C Reed ${ }^{5, e}$}

${ }^{1-5}$ School of Metallurgy and Materials, College of Engineering and Physical Sciences

The University of Birmingham, Edgbaston, Birmingham B15 2TT, United Kingdom

acXP864@bham.ac.uk, bj.gebelin@bham.ac.uk, cn.warnken@bham.ac.uk,

${ }^{d}$ r.w.broomfield@bham.ac.uk, ${ }^{e}$ r.reed@bham.ac.uk

Key words: Single Crystal Superalloys, Recrystallisation, Casting, Solidification

\begin{abstract}
During the manufacture of turbine blades from single crystal nickel-based superalloys by investment casting, recrystallisation can occur during solution heat treatment. The introduction of grain boundaries into a single crystal component is potentially detrimental to performance, and therefore manufacturing processes and/or component geometries should be chosen to prevent their occurrence. In this work, numerical models have been designed to enable a predictive capability for the factors influencing recrystallisation to be constructed. The root cause is plasticity on the microscale caused by differential thermal contraction of metal, mould and core; when the plastic deformation is sufficient, recrystallisation can take place subsequently. The models take various forms. First, one-dimensional models based upon static equilibrium have been produced - our calculations indicate that plastic strain is likely to take place in two temperature regimes: by creep between $1150^{\circ} \mathrm{C}$ and $1000^{\circ} \mathrm{C}$ and by tensile (time-independent) strain below $650^{\circ} \mathrm{C}$. The idea of a strain-based criterion for recrystallisation is then proposed. Second, more sophisticated threedimensional calculations based upon the finite element method are carried out. Our predictions are compared critically with experimental information.
\end{abstract}

\section{Introduction}

Currently, turbine manufacturers are facing unprecedented economic, societal and legislative pressures to make gas turbines - which are used for electricity generation and jet propulsion - fuel efficient, less polluting and even more reliable [1]. Advanced compositions of single crystal superalloys are making a contribution to this, as are thermal barrier coating systems which have been adopted widely. However, the designs of turbine blade geometries are also becoming increasingly sophisticated, to promote their aerodynamic performance and to allow cooling air to be used for greater cooling efficiency [2]. For the casting engineering, this situation presents significant challenges because significant quantities of turbine blades must be produced by the foundry, each of which must pass stringent tests to confirm that dimensional tolerances are met and that metallurgical defects are absent.

Recrystallised grains in the turbine blades designed to be used in single crystal form are one example. Recrystallisation can take place on both internal and external surfaces [3-8] of a casting, usually during the heat treatment which is required to remove the microsegregation inherited from casting; its occurrence cannot be tolerated since grain boundaries are then introduced. The root cause is believed to be differential thermal contraction of the superalloy, mould and core; because the thermal expansion coefficients of these differ markedly, thermal stresses are then generated which are sufficient to cause plastic deformation. Where plastic strain is accumulated and it exceeds a critical limit, recrystallised grains occur. The probability of the occurrence of this effect depends upon the alloy used, the shell and core systems employed as well as the details of the processing chosen for both casting and subsequent solution heat treatment.

At the present time, more quantitative information about the phenomenon of recrystallisation in single crystal superalloys is needed, particularly its dependence on accumulated plastic strain levels [9-12]. In addition, knowledge of the critical temperature at which the plastic strain is introduced would be useful for modelling purposes [13,14]. For this reason, in the present paper numerical modelling is carried out to analyse the mechanical response of the casting during 
solidification and subsequent cooling. In the first instance, to simplify the calculations a single crystal bar is modelled analytically in 1D - consistent with the solidification direction $<001>-$, with a number of different simplifications for the properties of the shell and core, and mechanical behaviour of the superalloy. Results from these calculations are compared with more sophisticated three-dimensional calculations carried out using finite element analysis [15-17].

\section{Background}

Based upon the theory of one-dimensional linear isotropic thermal-elasticity, the stress and strain behaviour of a superalloy in the solidification direction is

$$
\sigma^{\mathrm{el}}=\mathrm{E} \varepsilon^{\mathrm{el}}
$$

where $\sigma^{\mathrm{el}}, \mathrm{E}$ and $\varepsilon^{\mathrm{el}}$ are the elastic stress, Young's Modulus and elastic strain, respectively. The first assumption made here is that the system of superalloy/shell is a closed system - thus there is no external force applied; consequently, the thermal contraction strain must be perfectly distributed between the elastic, plastic (time-independent plastic deformation) and creep (time-dependent plastic deformation) strains. This means that the strains must sum to zero according to

$$
\sum \varepsilon=\varepsilon^{\mathrm{el}}+\varepsilon^{\mathrm{pl}}+\varepsilon^{\mathrm{cr}}+\varepsilon^{\mathrm{th}}=0,
$$

where $\varepsilon^{\mathrm{pl}}, \varepsilon^{\mathrm{cr}}$ and $\varepsilon^{\text {th }}$ are plastic, creep and thermal contraction strains, respectively. An isotropic hardening criterion for plastic strain is defined via the yield stress, $\sigma_{\mathrm{y}}$, ultimate yield stress, $\sigma_{\infty}$, and hardening exponent, $\mathrm{H}=0.3$, as follows

$$
\sigma=\sigma_{\infty}+\left(\sigma_{\mathrm{y}}-\sigma_{\infty}\right) \exp \left(-H \varepsilon^{\mathrm{pl}}\right) \text {. }
$$

Regarding the time-dependent plastic deformation, we can define the creep rate from Norton's creep equation:

$$
\frac{\mathrm{d} \varepsilon^{\mathrm{cr}}}{\mathrm{dt}} \equiv \dot{\varepsilon}^{\mathrm{cr}}=\frac{1}{\eta} \exp \left(-\frac{\mathrm{Q}}{\mathrm{RT}}\right) \sigma^{\mathrm{n}}
$$

where $\eta=2.2 \times 10^{-6} \mathrm{~s}$ is the viscosity parameter used here which is assumed to be constant, $\mathrm{Q}$ is an activation energy which is taken to be $870 \mathrm{~kJ} / \mathrm{mol}, \mathrm{R}$ is the gas constant, $\mathrm{T}$ is temperature and $\mathrm{n}$ is stress exponent which is taken to be 10 . By integrating over time, the creep strain can be written as

$$
\varepsilon^{\mathrm{cr}}=\int_{\mathrm{t}_{\mathrm{i}}}^{\mathrm{t}_{\mathrm{f}}} \dot{\varepsilon}^{\mathrm{cr}} \mathrm{dt},
$$

where $t_{i}$ and $t_{f}$ are initial and final time, respectively. As for the thermal contraction strain, $\varepsilon^{\text {th }}$, it is proportional to the temperature via the thermal expansion coefficient, $\alpha$, which is

$$
\varepsilon^{\text {th }}=\alpha \Delta \mathrm{T}=\alpha\left(\mathrm{T}-\mathrm{T}_{\mathrm{ref}}\right)
$$

where $\mathrm{T}_{\text {ref }}$ is reference temperature. By making use of the Eq.2, Eq.3, Eq.4 and Eq.5, Eq.1 becomes $\sigma^{\mathrm{el}}=\mathrm{E}\left(-\varepsilon^{\mathrm{pl}}-\varepsilon^{\mathrm{cr}}-\varepsilon^{\text {th }}\right)$.

In this way, the stress and strains built-up within the superalloy bar can be calculated. The parameters used in the modelling are chosen to be consistent with the second generation single crystal superalloy CMSX-4; other data used in the calculations are given in [18-22].

\section{Model Descriptions}

Thermo-mechanical modelling of a one-dimensional bar of CMSX-4 under rigid constraints is carried out. It is assumed that the superalloy and shell adhere together during solidification and subsequent cooling to room temperature. The nominal cooling rate used for the calculation is $50^{\circ} \mathrm{C} / \mathrm{min}$ or $0.83^{\circ} \mathrm{C} / \mathrm{s}$. Consistent with the equilibrium condition, any force other than that arising from shrinkage is neglected. Isotropic linear elasticity has been used for this work. 


\section{The behaviour of the superalloy}

The behaviour of the superalloy is analysed assuming that: (i) the shell system is perfectly rigid, and (ii) the shell system is elastic. For the metal, three models are employed:

\section{- Linear elastic model}

Following Eq.2, only two strains - elastic and thermal - are used. The others are set to zero. The elastic strain in this scheme is derived from the thermal strain. Moreover, the elastic stress can be obtained from the elastic strain and the Young's Modulus.

\section{- Elastoplastic model}

Here, it is assumed that three strains exist within the superalloy: namely, thermal, elastic and plastic strains; creep is ignored. The thermal strain will be partitioned into elastic and plastic portions. When an isotropic hardening criterion is used consistent with Eq.3, the elastic stress predicted is different from that from the linear elastic model.

- Elasto-viscoplastic model

The elasto-viscoplastic model is considered; this is expected to be a more rigorous treatment for calculating the stress and strain in the superalloy. This is because the elastic, plastic and creep strains are taken into account and decoupled.

\section{The plasticity approximation}

Plasticity within the superalloy can be calculated by making use of Eq.3. By assuming that the plastic strain accumulated in the superalloy is of order $10^{-3}$, the exponential term in Eq.3 can be approximated by using Taylor's Series:

$$
\exp (\mathrm{x})=\sum_{\mathrm{n}=0}^{\mathrm{N}} \frac{1}{\mathrm{n} !} \mathrm{x}^{\mathrm{n}}
$$

The first order term is to be kept. When a perfectly rigid shell is assumed, the plastic strain for the superalloy can then be determined. When the elasto-visco-plastic model is employed, the plastic strain can be expressed by

$$
\varepsilon^{\mathrm{pl}}=\frac{\sigma_{\mathrm{y}}+\mathrm{E} \varepsilon^{\mathrm{th}}+\mathrm{E} \varepsilon^{\mathrm{cr}}}{\mathrm{H}\left(\sigma_{\mathrm{y}}-\sigma_{\infty}\right)} .
$$

When the elastoplastic model is used, $\varepsilon^{\text {th }}$ is set to zero.

\section{Results and Discussion}

The results of the analytical modelling are compared with predictions from the ProCAST finite element tool. The situation corresponding to a cylindrical, constrained bar have been modelled using ProCAST, with care being taken to ensure that the mesh is sufficiently fine for the results to be independent of element number. The results quoted correspond to nodes at the centre of the bar.

\section{- Linear elastic model}

If purely elastic properties are assumed, the thermal stress expected from the cooling of a CMSX-4 bar under rigid constraint can be estimated. Fig.1(a) shows the strain evolution with temperature in the one-dimensional constrained bar; namely, thermal and elastic strain. The elastic strain increases monotonically in an approximately linearly fashion. The thermal strain is of opposite sign; thus the sum of the two strains is zero. The calculated stress is consistent with the strains given in Fig. 1(b).
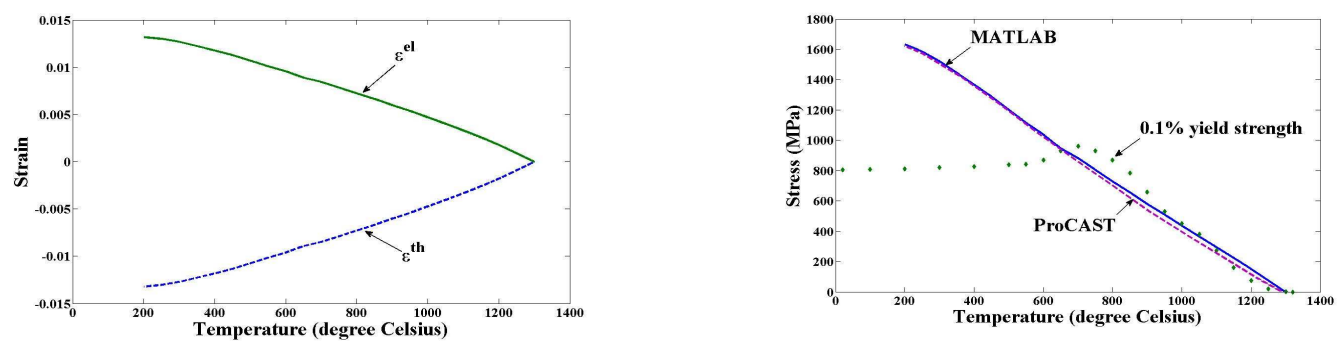

The stresses calculated from MATLAB and ProCAST are consistent with each other.
(a) Strain built-up in the bar
(b) Calculated stress

Fig.1 Linear elastic model results from MATLAB and ProCAST. 


\section{- Elastoplastic model}

The elastoplastic model takes the plastic straining effect into account. It can be seen in Fig. 2(a) that plastic deformation occurs from $1300^{\circ} \mathrm{C}$ to roughly $1100^{\circ} \mathrm{C}$, and then the total accumulated plastic strain stays constant. This is an elastic region. When the temperature fall below $650^{\circ} \mathrm{C}$, there is a dramatic increase in plastic strain; at this stage the elastic strain declines continuously. Clearly, the stress level predicted is high - higher than the yield stress of the material at low temperatures, indicating that plastic yielding is a strong possibility. The stress results given in Fig. 2(b) illustrate the variation of calculated stress

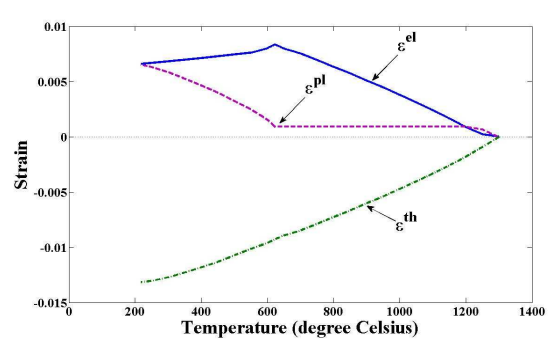

(a) Strain diagram from elasto-plastic model

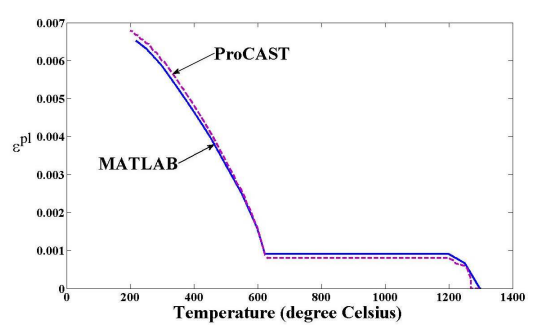

(c) Plastic strains from MATLAB and ProCAST

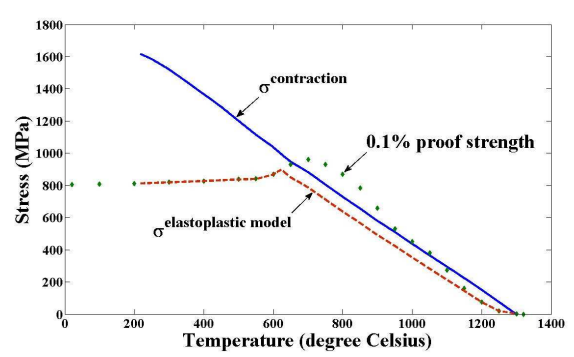

(b) Calculated stresses

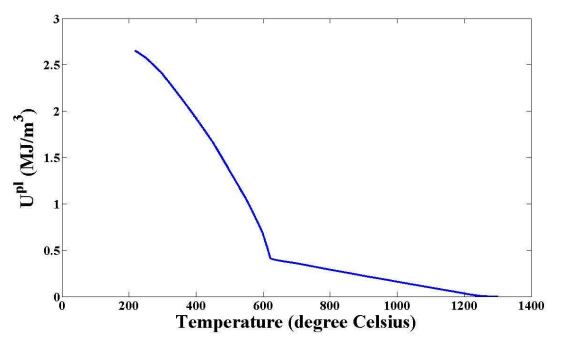

(d) Calculated plastic strain energy

Fig. 2 Elasto-plastic model simulated by MATLAB and ProCAST.

and yield stress with temperature. Above $1100^{\circ} \mathrm{C}$, the predicted stress is slightly higher than the experimental $0.1 \%$ proof strength, but at lower temperature it falls below it. Below $650^{\circ} \mathrm{C}$, the thermal stress is again greater than the yield stress. Thus, in the absence of stress-relieving mechanisms and certainly if stress concentration effects are present, plasticity must occur.

The effective plastic strain expected in a simple circular cylindrical test bar $(12 \mathrm{~mm}$ diameter) at its centre simulated by ProCAST is compared with the one-dimensional model in Fig. 2(c). It is found that the results are consistent with each other. This implies that the one-dimensional model is representative of the situation assumed. Plastic strain energy is also calculated so as to see the level of the energy or force created by the plastic deformation, see Fig. 2(d). According to driving force due to plastic deformation, $\mathrm{F}=\mathrm{Gb}^{2} \rho$, it is assumed that the plastic strain energy, $\mathrm{U}^{\mathrm{pl}}$, can be expressed in terms of

$$
\mathrm{U}^{\mathrm{pl}}=\int_{0}^{\varepsilon^{\mathrm{pl}}} \sigma \mathrm{d} \varepsilon=\sigma \varepsilon^{\mathrm{pl}} .
$$

It is interesting to make quantitative estimates. For pure nickel, $\mathrm{G}$ is $76 \mathrm{GPa}, \mathrm{b}$ is $0.25 \mathrm{~nm}$ and assuming $\rho$ is $10^{14} \mathrm{~m}^{-2}$ the energy is equal to $0.475 \mathrm{MJ} / \mathrm{m}^{3}$, which is consistent with the driving force for recrystallisation in pure nickel at room temperature. The values should be somewhat higher than this in practice because of the higher shear modulus of the nickel-base superalloys, and because the dislocation density induced is likely to be greater than that assumed. 
- Elasto-viscoplastic model

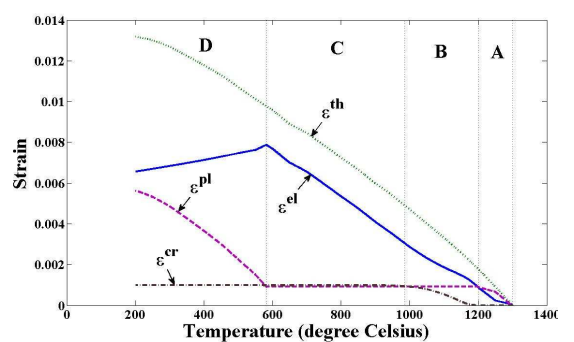

(a) Strain diagram

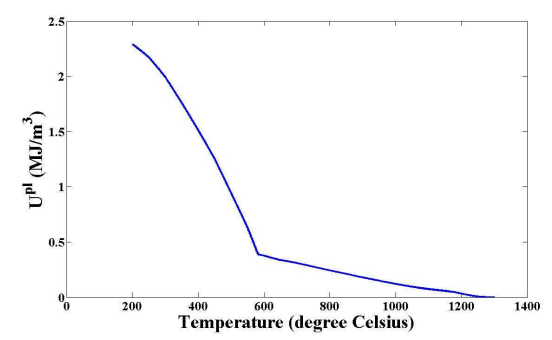

(d) Predicted plastic strain energy

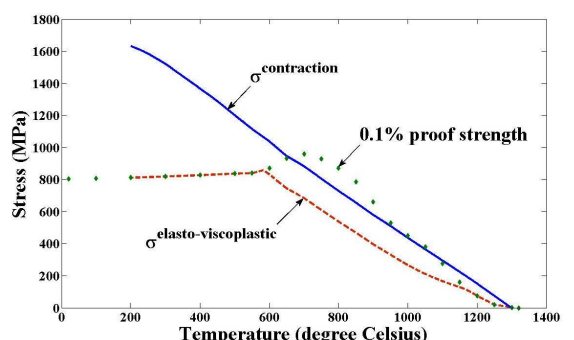

(b) Calculated stress

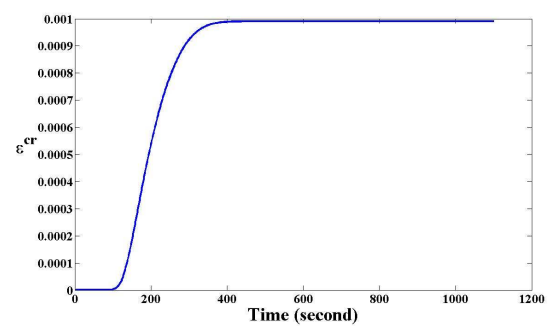

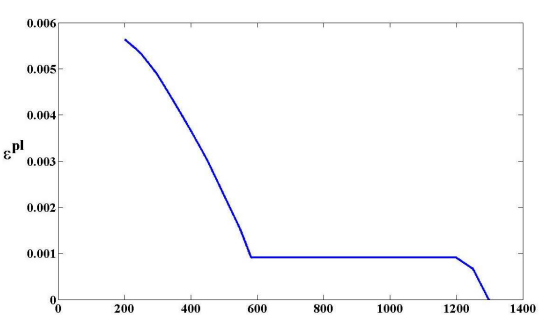

(c) Predicted plastic strain

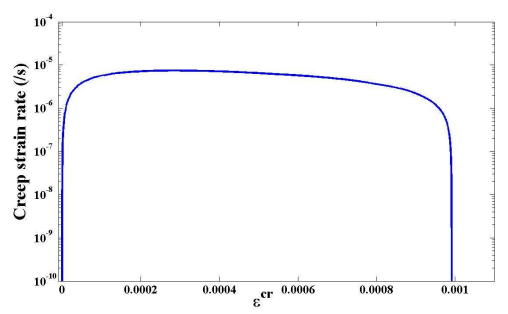

Fig.3 Elasto-viscoplastic model simulated by MATLAB

Fig.3 shows results obtained from the elasto-viscoplastic model. Strains generated within the superalloy are illustrated in Fig.3(a). It has become clear that there are at least four zones in which differing behaviour is expected. In zone A, the superalloy starts yielding during the very early stages of the cooling process (between 1300 to $1200^{\circ} \mathrm{C}$ ). This is because the proof strength is very low at these temperatures as shown in Fig.3(b), and the thermal contraction stress exceeds it. In zone $\mathrm{B}$, the proof stress increases rapidly to exceed the thermal stress; consequently, accumulation of plastic strain ceases. However, the temperature is still sufficiently high for creep strain to be accumulated from 1200 to $1000^{\circ} \mathrm{C}$. Thus creep and elastic deformation occurs in this region. In zone $\mathrm{C}$ - from roughly 1000 to $600^{\circ} \mathrm{C}$ - the proof strength is above thermal stress, so there is only elastic deformation in this zone. Finally, below $600^{\circ} \mathrm{C}$ in zone D, the proof strength is again much lower than the contraction stress; as a result, plastic straining is predicted to occur and to continue up to $0.6 \%$. The superalloy work hardening is consistent with the isotropic hardening criteria. During this stage, the stress built-up in the superalloy decreases but is slightly higher than the $0.1 \%$ yield strength. This region has a significant increase in plastic strain and a decrease of elastic strain.

The levels of plastic strain and plastic strain energy are plotted in Fig.3(c) and Fig. 3(d), respectively. It can be seen that the temperature at which the plastic strain is switched on changes from roughly $650^{\circ} \mathrm{C}$ to below $600^{\circ} \mathrm{C}$. The variation of creep strain with time and the variation of creep strain rate with creep strain has been given in Fig.3(e) and Fig.3(f), respectively.

\section{Summary and Conclusions}

One-dimensional modelling of a constrained bar of the superalloy CMSX-4 has been executed under three assumptions: linear elastic, elasto-plastic and elasto-viscoplastic. It is demonstrated that for the superalloy sitting within a perfectly rigid shell, plasticity is expected to occur in two regimes: namely, by creep strain between $1150^{\circ} \mathrm{C}$ and $1000^{\circ} \mathrm{C}$ and tensile strain at below $650^{\circ} \mathrm{C}$.

\section{Acknowledgments}

The author thanks the Engineering and Physical Science Research Council (EPSRC) and RollsRoyce plc for funding his studies via a Dorothy Hodgkin Postgraduate Award (DHPA). Moreover, the author would like to extend special thanks to Paul Brown and Dr Duncan Putman from RollsRoyce plc, and Dr Rajab Said and Jez Smith of the ESI Group. 


\section{References}

[1] D.C. Cox, B. Roebuck, C.M.F. Rae, and R.C. Reed: Materials Science and Technology Vol. 19 (2003), p. 440-446

[2] R.C. Reed: The Superalloys: Fundamentals and Applications. Cambridge University Press, Cambridge (2006).

[3] P.R. Rios, F. S. Jr, H.R.Z. Sandim, R.L. Plaut, and A.F. Padiha: Materials Research Vol. 8 (2005), p. 225-238

[4] R.D. Doherty, D.A. Hughes, F.J. Humphreys, J.J. Jonas, D. Juul Jensen, M.E. Kass- ner, W.E. King, T.R. McNelley, H.J. McQueen, and A.D. Rollett: Materials Science and Engineering, Vol. A238 (1997), p.219-274

[5] J.G. Byrne: Recovery, Recrystallisation and grain growth. The MacMillan Company, New York (1965).

[6] P. Cotterill and P.R. Mould: Recrystallisation and grain growth in metals. Surrey University Press, London (1976).

[7] F.J. Humphreys and M. Hatherly: Recrystallisation and related annealing phenomena. Elsevier Science Ltd., Oxford (1995).

[8] S.S. Gorelik: Recrystallization in metals and alloys. Moscow: MIR Publishers, 1981.

[9] Sir Alan Cottrell: An introduction to metallurgy. Edward Arnold (Publishers) Ltd, Cambridge, 2nd edition (1975).

[10] B. N. J. Persson: Physical Review B, Vol. 61(2000), p.5949-5966

[11] R.W.K. Honeycombe: The plastic deformation of metals. Edward Arnold Publishers. Ltd., London, 2nd edition (1984).

[12] A.J. Fletcher: Thermal stress and strain generation in heat treatment. Elsevier Science Publishers. Ltd., Essex (1989).

[13] G.E. Dieter: Mechanical Metallurgy. Materials Science and Engineering Series. McGraw-Hill, Singapore, 3rd edition (1986).

[14] V.E. Saouma: Advanced Mechanics of Materials. University of Colorado, Boulder, (2002).

[15] T.R. Chandrupatla and A.D. Belegundu. Introduction to Finite Elements in Engineering. Prentice hall, New Jersey (1991).

[16] ESI Group: ProCAST 2009.0 User's Manual Volume 1. (2009).

[17] ESI Group: ProCAST 2009.0 User's Manual Volume 2. (2009).

[18] R.W. Broomfield. Review of creep data on As-cast RR3010 and CMSX-4, generated by the National Physical Laboratory. Technical report, The University of Birmingham, March 2009.

[19] B. Roebuck and R. Morrell: Elevated temperature mechanical and physical property tests on single crystal Ni alloys. CMMT(D) 269, National Physical Laboratory, (2000).

[20] R. Morrell, J. M. Cox, B. Monaghan, and L. Chapman. Property characterisation of core and mould ceramics for directional solidification of turbine blade alloys. CMMT(D) 259, National Physical Laboratory, (2001).

[21] R. Morrell and L. Chapman. Thermal and mechanical properties of a casting mould material. Technical Report 07110082/1, National Physical Laboratory, (2008).

[22] R. Morrell and L. Chapman. Thermal and mechanical properties of a casting mould material. Technical Report E07110005/1, National Physical Laboratory, (2008). 